\title{
The prevalence and risk factors of school absenteeism due to premenstrual disorders in Japanese high school students-a school-based cross-sectional study
}

\author{
Mari Tadakawa ${ }^{1}$, Takashi Takeda ${ }^{1,2^{*}}$, Yasutake Monma ${ }^{3}$, Shoko Koga ${ }^{4}$ and Nobuo Yaegashi ${ }^{1}$
}

\begin{abstract}
Background: Premenstrual disorders such as premenstrual syndrome (PMS) and premenstrual dysphoric disorder (PMDD) interfere with the daily lives of adolescents. The causes of PMS and PMDD are unknown, but lifestyle habits, such as regular exercise and taste preference are known to be associated. This study was conducted to investigate how premenstrual symptoms affect the school life in Japanese high school students and whether there was a risk factor for school absenteeism that is dependent on the types of premenstrual symptoms or lifestyle habits.

Methods: A school-based survey was conducted in Sendai, an industrial city in Japan. A total of 901 girls aged 15-19 with regular menstrual cycles were assessed using the self-reporting premenstrual symptoms questionnaire (PSQ) and questions regarding school absence, taste preference, and exercise. We classified the girls into 'no/mild PMS', 'moderate-to-severe PMS' and 'PMDD' according to the PSQ. The girls were classified into the 'absent' group if they were absent for more than 1 day per month. We used multivariate logistic analysis to examine the risk factors for school absenteeism.

Results: The rates of 'moderate-to-severe PMS' and 'PMDD' were 9.9 and $3.1 \%$, respectively. A total of 107 girls $(11.9 \%)$ were classified into the 'absent' group. Significant differences were observed in the prevalence of all premenstrual symptoms $(p<0.001)$, 'age' ( $p<0.001)$, 'a preference for salty food' ( $p=0.001)$, and 'lack of regular exercise' ( $p=0.03$ ) between the 'absent' and 'non-absent' groups. Multivariate analysis revealed that premenstrual symptoms such as 'insomnia or hypersomnia' (odds ratio [OR] 2.27, $95 \%$ confidence interval [Cl]: 1.46-4.17) and 'physical symptoms' (OR 2.24, 95 \% Cl: 1.37-3.66), 'reduced social life activities' (OR 2.71, 95 \% Cl 1.31-5.59), and 'a preference for salty food' (OR 1.89, $95 \%$ Cl: 1.20-2.98) were risk factors for school absenteeism.

Conclusions: One in nine Japanese female high school students were absent from school due to premenstrual symptoms. Physical premenstrual symptoms and lifestyles, such as a preference for salty food and a lack of regular exercise, were identified as risk factors for school absenteeism.
\end{abstract}

Keywords: Premenstrual syndrome, Premenstrual dysphoric disorder, Japanese high school students, Adolescence, School absenteeism, Salty food, Exercise

\footnotetext{
* Correspondence: take@med.kindai.ac.jp

'Department of Obstetrics and Gynecology, Tohoku University Graduate

School of Medicine, 1-1 Seiryocho, Aobaku, Sendai, Japan

2Division of Women's Health, Research Institute of Traditional Asian Medicine,

Kindai University School of Medicine, 377-2 Ohno-Higasi, Osaka-Sayama,

Osaka 589-8511, Japan

Full list of author information is available at the end of the article
}

Biomed Central

(C) 2016 Tadakawa et al. Open Access This article is distributed under the terms of the Creative Commons Attribution 4.0 International License (http://creativecommons.org/licenses/by/4.0/), which permits unrestricted use, distribution, and reproduction in any medium, provided you give appropriate credit to the original author(s) and the source, provide a link to the Creative Commons license, and indicate if changes were made. The Creative Commons Public Domain Dedication waiver (http://creativecommons.org/publicdomain/zero/1.0/) applies to the data made available in this article, unless otherwise stated. 


\section{Background}

Premenstrual syndrome (PMS) is characterized by emotional, behavioral, and physical symptoms that occur during the late luteal phase of the menstrual cycle and are relieved after the onset of menstruation. Epidemiological studies have reported that $5-8 \%$ of reproductive age women exhibit moderate-to-severe premenstrual symptoms that interfere with their daily activities [1]. Other studies have suggested that more than $20 \%$ of fertile women have premenstrual symptoms that cannot be clinically ignored [2]. A severe form of PMS has been classified as premenstrual dysphoric disorder (PMDD) according to the Diagnostic and Statistical Manual of Mental Disorders, fifth edition (DSM-5) [3]. Such premenstrual disorders were originally considered to begin in the early twenties in women [4]. However, recent studies have demonstrated that they start earlier in the teenage years $[5,6]$.

Adolescence is a unique time in human development that produces significant psychological and physiological changes. Dysmenorrhea is a known health concern in adolescence. In our previous study, we reported a correlation between dysmenorrhea and PMS/PMDD in Japanese high school students [7]. We also showed that the frequencies of 'moderate-to-severe PMS' and 'PMDD' in Japanese high school students were 11.6 and $2.6 \%$ higher, respectively, than that of adult women (5.3 and $1.2 \%$ ) [5]. These findings suggested that PMS and PMDD are major problems that affect the daily lives of adolescents, possibly to a larger extent than adults. Although studies have been conducted to investigate the prevalence of PMS and PMDD in adolescents [8-11], no study has ever revealed how premenstrual symptoms actually affect the school or social life of girls in adolescence.

The causes of PMS and PMDD have not yet been clearly elucidated, but they have been suggested to include hormonal changes, neurotransmitters, stress, and lifestyle habits [1, 4]. Lifestyle habits involve factors such as diet and exercise. The relationship between PMS and diet, for example, calcium intake [8, 12], caffeine-containing beverages [13, 14], and chocolate or sweets containing refined sugar [15], has been reported. Many studies demonstrated that exercise could be used as a treatment for premenstrual disorders [16, 17], and these findings indicated an association between PMS and lack of exercise.

The aim of this study was to investigate how premenstrual symptoms affect the school and social life of Japanese high school students, with a focus on its influence on school attendance. We also compared the types of premenstrual symptoms and lifestyle habits of 'absent' girls and 'non-absent' girls to determine the risk factors for school absenteeism.

\section{Methods}

This study was conducted in accordance with the principles outlined in the Declaration of Helsinki. Our Institutional Review Board at Tohoku University approved the study.

\section{Participants}

A school-based survey was conducted in October 2009 using a sample of Japanese female high school students who belonged to four public high schools in Sendai, an industrial city in Japan. We recruited students, aged 15-19, with regular menstrual cycles (22-35 days) who were able to provide informed consent.

We have already reported the preliminary data obtained from one of these schools [5].

\section{Questionnaire}

We used the Premenstrual Symptoms Questionnaire (PSQ), which was developed in our previous study [18], to screen for premenstrual symptoms. The PSQ translates the DSM-4 criteria [19] into a rating scale with degrees of severity described in Japanese and is essentially identical to the Premenstrual Symptoms Screening Tool [20]. The PSQ asked, "Within the last three months, have you experienced the following premenstrual symptoms starting during the week before menses and remitting a few days after the onset of menses?" The premenstrual symptoms listed in the PSQ were 'depressed mood,' 'anxiety or tension', 'tearful', 'anger or irritability', 'decreased interest in work, home, or social activities', 'difficulty concentrating,' 'fatigue or lack of energy', 'overeating or food cravings', 'insomnia or hypersomnia,' 'feeling overwhelmed', and 'physical symptoms such as tender breasts, feeling bloating, headache, joint or muscle pain, and weight gain'. The PSQ also asked whether such premenstrual symptoms interfered with 'work efficiency or productivity, home responsibility', 'social life activities', or 'relationships with coworkers or family'. The PSQ asked participants to rate the degree of severity of symptoms into four grades: 'not at all', 'mild,' 'moderate', and 'severe'. We classified participants into three groups: 'PMDD', 'moderate-to-severe PMS', and 'no/mild PMS' based on the criteria suggested by Steiner et al. [20], as used in our previous study. [18]. We defined the 'PMDD' girls as those who reported at least one of the four core symptoms ('depressed mood', 'anxiety or tension,' 'tearful', and 'anger or irritability') as severe and at least four additional symptoms (for a total of five) as moderate to severe. They also had to report that their symptoms interfered severely with their ability to function in at least one of three domains ('work efficiency or productivity, home responsibility', 'social life activities', 'relationship with coworkers or family'). The 'moderate-to-severe PMS' group, who marginally missed fulfilling the DSM criteria for the diagnosis of PMDD, 
were girls who reported at least one of the four core symptoms as moderate to severe and at least four additional symptoms as moderate to severe. They also reported that their symptoms interfered moderately or severely with their ability to function in at least one of the three domains listed above. In addition to the PSQ, we asked the participants whether they were absent from school due to the previously described premenstrual symptoms and the number of days per month they were absent in the last three months. We classified participants into the 'absent' group if they had more than 1 day of absence per month and the others into the 'non-absent' group, as defined in previous studies [21].

We also asked participants the following questions in order to establish their lifestyle habits, such as taste preferences and the extent of daily exercise: 'Do you regularly exercise (more than once per week)?, 'Do you prefer sweets?', 'Do you prefer salty food?', and 'Do you drink coffee?'. The questions were answered as either 'yes' or 'no'. The questionnaire consisted of 19 questions.

\section{Statistical analysis}

Data were analyzed statistically using the Statistical Analysis System 9.1 edition for WINDOWS (SAS Institute Inc, Cary, NC). We also used multivariate logistic analysis to examine the risk factors for school absenteeism. $P<0.05$ was adopted as the level of significance.

\section{Results}

A total of 1909 female students answered the questionnaire, with 1008 being excluded from the analysis because of incomplete data. Therefore, we analyzed the data of 901 girls aged 15-19 years. The background characteristics of the participants are described in Table 1. Eighty-nine participants $(9.9 \%)$ were classified as having 'moderateto-severe PMS' and 28 (3.1 \%) as having 'PMDD'. The prevalence of each premenstrual symptom is shown in Table 2. More than half of the participants had 'anxiety or tension' (66.7\%), 'anger or irritability' (64.0\%), 'difficulty concentrating' (59.5\%), 'fatigue or lack of energy' (70.9\%), 'overeating or food cravings' (52.8 \%), and 'physical symptoms' (60.9 \%). Premenstrual symptoms impaired 'work efficiency or productivity, home responsibility' in $50.7 \%$

Table 1 Background characteristics of the participants $(N=901)$

\begin{tabular}{ll}
\hline Characteristics & \\
\hline Age, years \pm SD & $17.0 \pm 1.0$ \\
Regular exercise habits, no. (\%) & $490(54.4)$ \\
Prefer sweets, no. (\%) & $712(79.0)$ \\
Prefer salty food, no. (\%) & $351(39.0)$ \\
Drink coffee, no. (\%) & $662(73.2)$
\end{tabular}

$S D$ standard deviation of the participants, 'social life activities' in $23.3 \%$, and 'relationships with coworkers or family' in $24.0 \%$.

A total of 107 girls (11.9\%) were classified into the 'absent' group, which demonstrated that premenstrual symptoms affected school attendance. Table 3 shows the number of days of absence: 94 participants were absent for 1-3 days, 8 participants for 4-6 days, and 5 participants for 7-10 days. No participants were absent for more than 10 days. The mean number of absent days for all participants was 0.3 days per month, with 0.2 days for 'no/mild PMS', 0.9 days for 'moderate-to-severe PMS', and 0.9 days for 'PMDD'. Although the prevalence of absent girls increased based on the severity of PMS, 64 (8.2\%) participants with 'no/mild PMS' were classified into the 'absent' group, which revealed that premenstrual symptoms are associated with school absenteeism not only in over-moderate PMS and PMDD groups, but also in participants with 'no/mild PMS'.

Significant differences were observed in the prevalence of all premenstrual symptoms $(p<0.001)$ between the 'absent' group and the 'non-absent' group. Among background and lifestyle risk factors, 'age' $(p<0.001)$, 'regular exercise habits' $(p=0.03)$ and 'a preference for salty food' $(p=0.001)$ were also significantly different (Table 4$)$. Multivariate logistic analysis revealed that 'a preference for salty food' (odds ratio [OR] 1.89, $95 \%$ confidence interval [CI]: $1.20-2.98)$ and premenstrual symptoms such as 'insomnia or hypersomnia' (OR 2.27, 95 \% CI: 1.32-3.38), 'physical symptoms, such as tender breasts, feeling bloating, headache, joint or muscle pain, and weight gain' (OR 2.24, 95 \% CI: 1.35-3.67) and 'reduced social life activities' (OR 2.71, 95 \% CI: 2.71-5.59) increased the risk of school absence (Table 5).

\section{Discussion}

Apart from our previous study [5], epidemiological studies have revealed a high prevalence of PMS and PMDD in adolescents: Steiner et al. [9] reported the prevalences as 8.3 \% for PMDD and 21.3 \% for severe PMS, whereas Drosdzol et al. [11] reported them as $4.17 \%$ for PMDD and $76.39 \%$ for PMS. In the present study, the prevalences of 'PMDD' and 'moderate-to-severe PMS' were 3.1 and $9.9 \%$, respectively. Moreover, Delara et al. [22] reported a significantly poor health-related quality of life in Iranian girls with PMDD using an index score from the Short Form Health Survey. However, to the best of our knowledge, no study has established the actual effects of premenstrual disorders on the school or social lives of adolescents.

Here, we present the first school-based survey to show how premenstrual disorders affect the school and daily lives of adolescents. According to the results obtained in this study, $11.9 \%$ of Japanese high school students were absent from school due to premenstrual symptoms and 
Table 2 Prevalence rates of premenstrual symptoms and induced interference with work, usual activities, or relationships ( $N=901)$

\begin{tabular}{|c|c|c|c|c|}
\hline Symptoms & Not at all & Mild & Moderate & Severe \\
\hline \multicolumn{5}{|l|}{ Premenstrual symptoms, no. (\%) } \\
\hline Depressed mood & $459(50.9)$ & $262(29.1)$ & $136(15.1)$ & $44(4.9)$ \\
\hline Anxiety or tension & $300(33.3)$ & $324(36.0)$ & $196(21.8)$ & $81(9.0)$ \\
\hline Tearful & $490(54.4)$ & $217(24.1)$ & $130(14.4)$ & $64(7.1)$ \\
\hline Anger or irritability & $324(36.0)$ & $304(33.7)$ & $196(21.8)$ & $77(8.5)$ \\
\hline Decreased interest in work, home, or social activities & $608(67.5)$ & $198(22.0)$ & $77(8.5)$ & $18(2.0)$ \\
\hline Difficulty concentrating & $365(40.5)$ & $370(41.1)$ & $129(14.3)$ & $37(4.1)$ \\
\hline Fatigue or lack of energy & $262(29.1)$ & $340(37.7)$ & $226(25.1)$ & $73(8.1)$ \\
\hline Overeating or food cravings & $425(47.2)$ & $243(27.0)$ & $155(17.2)$ & $78(8.7)$ \\
\hline Insomnia or hypersomnia & $461(51.2)$ & $243(27.0)$ & $133(14.8)$ & $64(7.1)$ \\
\hline Feeling overwhelmed & $622(69.0)$ & $195(21.6)$ & $63(7.0)$ & $21(2.3)$ \\
\hline Physical symptoms & $352(39.1)$ & $283(31.4)$ & $182(20.2)$ & $84(9.3)$ \\
\hline \multicolumn{5}{|l|}{ Interference with work, usual activities, or relationships, no. (\%) } \\
\hline Work efficiency or productivity, home responsibility & $445(49.3)$ & $316(35.1)$ & $110(12.2)$ & $30(3.3)$ \\
\hline Social life activities & $691(76.7)$ & $148(16.4)$ & $44(4.9)$ & $18(2.0)$ \\
\hline Relationships with coworkers or family & $685(76.0)$ & $165(18.3)$ & $41(4.6)$ & $10(1.1)$ \\
\hline
\end{tabular}

up to $5.2 \%$ of girls were absent for 2 days or more per month. This result supports the recent findings $[9,11,22]$ of studies in which premenstrual disorders were shown to be major problems in adolescence that markedly interfered with school and social activities. Significant differences were observed in the prevalence of all premenstrual symptoms between the 'absent' group and the 'non-absent' group in the present study. 'Absent' girls consisted not only of participants that were classified with 'moderateto-severe PMS' or 'PMDD', but also of a high rate of participants with 'no/mild PMS'. This result indicated that premenstrual symptoms may lead to school absenteeism in any girl that has menses and ovulates. An analysis of factors interfering with work, activities, and relationships showed that 'reduced social life activities' was a risk factor for school absenteeism, conversely indicating that girls in the 'absent' group were having problems not only in their school lives but also in other social activities, such as hobbies or after-school activities.

A limitation of our study was that this was a retrospective, self-reporting design. Therefore, recall bias should be considered. Because menstruation-related symptoms differ to some extent in each cycle, some participants may have also had difficulties in accurately answering the questions. This may explain the high frequency of incomplete data noted in the present study. The low percentage of response (901/1909) should also be considered as a limitation. However, the rate of PMS severity was similar to that in our previous studies (prevalence of 'moderate-to-severe PMS' and 'PMDD' were 11.8 and $2.6 \%$ in 2010 [5], and 11.3 and $3.2 \%$ in 2012 [7], respectively). Therefore, we think that the data in the present study are reliable. To exclude bias due to educational levels, we recruited participants from four high schools of various levels. However, we should include high schools from other areas to examine the true prevalence of premenstrual symptoms and its effect on Japanese adolescent students.

The causes of PMS and PMDD have not yet been clarified but are believed to include lifestyle factors, such as diet and exercise. Previous studies have suggested associations between PMS and caffeine intake [13, 14], chocolate consumption [15], and exercise [16, 17]. However, few of

Table 3 Days of absence per month due to premenstrual symptoms

\begin{tabular}{lllll}
\hline Absent & Total & No/mild PMS & Moderate-to-severe PMS & $n=28$ \\
\hline Yes & $N=901$ & $n=784$ & $n=89$ & $12(42.9)$ \\
$1-3$ day(s) & $107(11.9)$ & $64(8.2)$ & $31(34.8)$ & $10(35.7)$ \\
$4-6$ days & $94(10.4)$ & $58(7.4)$ & $26(29.2)$ & $2(7.1)$ \\
$7-10$ days & $8(0.9)$ & $1(0.6)$ & $1(1.1)$ & $0(0.0)$ \\
\hline
\end{tabular}


Table 4 Comparison between the 'absent' group and 'non-absent' group

\begin{tabular}{|c|c|c|c|}
\hline & Absent group $n=107$ & Non-absent group $n=794$ & $P$-value \\
\hline \multicolumn{4}{|l|}{ Background and lifestyle risk factors, no. (\%) } \\
\hline Age, years $\pm S D$ & $17.0 \pm 1.0$ & $16.6 \pm 1.0$ & $<0.001^{*}$ \\
\hline Regular exercise habits & $47(43.9)$ & $443(55.8)$ & $0.03^{*}$ \\
\hline Prefer sweets & $85(79.4)$ & $627(79.0)$ & 1.000 \\
\hline Prefer salty food & $58(54.2)$ & $293(36.9)$ & $0.001^{*}$ \\
\hline Drink coffee & $36(33.6)$ & $204(25.7)$ & 0.09 \\
\hline \multicolumn{4}{|l|}{ Premenstrual symptoms (over moderate severity), no. (\%) } \\
\hline Depressed mood & $47(43.9)$ & $133(16.8)$ & $<0.001^{*}$ \\
\hline Anxiety or tension & $62(57.9)$ & $215(27.1)$ & $<0.001^{*}$ \\
\hline Tearful & $53(49.5)$ & $141(17.8)$ & $<0.001^{*}$ \\
\hline Anger or irritability & $64(59.8)$ & $209(26.3)$ & $<0.001^{*}$ \\
\hline Decreased interest in work, home, or social activities & $32(29.9)$ & $63(7.9)$ & $<0.001^{*}$ \\
\hline Difficulty concentrating & $41(38.3)$ & $125(15.7)$ & $<0.001^{*}$ \\
\hline Fatigue or lack of energy & $71(66.4)$ & $228(28.7)$ & $<0.001^{*}$ \\
\hline Overeating or food cravings & $48(44.9)$ & $185(23.3)$ & $<0.001^{*}$ \\
\hline Insomnia or hypersomnia & $57(53.3)$ & $140(17.6)$ & $<0.001^{*}$ \\
\hline Feeling overwhelmed & $27(25.2)$ & $57(7.2)$ & $<0.001^{*}$ \\
\hline Physical symptoms & $64(59.8)$ & $202(25.4)$ & $<0.001^{*}$ \\
\hline \multicolumn{4}{|c|}{ Induced interference with work, usual activities, or relationships (over moderate severity), no. (\%) } \\
\hline Work efficiency or productivity, home responsibility & $41(38.3)$ & $99(12.5)$ & $<0.001^{*}$ \\
\hline Social life activities & $28(26.2)$ & $34(4.3)$ & $<0.001^{*}$ \\
\hline Relationships with coworkers or family & $23(21.5)$ & $28(3.5)$ & $<0.001^{*}$ \\
\hline
\end{tabular}

*Statistically significant $(p$ value $<0.05)$

$S D$ standard deviation

these have been evaluated systematically. In our study, no correlations were observed between school absence and 'a preference for sweets' $(p=1.00)$ or 'drinking coffee' $(p=0.09)$, but a correlation was observed for 'regular exercise habits' ( $p=0.03)$ and 'a preference for salty food' $(p=0.001)$. The association with the habit of regular exercise supports the findings of previous studies [16, 17]. Exercise may improve premenstrual symptoms mainly in emotional and physical conditions by increasing betaendorphin levels and physical well-being [22]. Previous studies have reported the effectiveness of exercise towards physical symptoms, including breast tenderness and fluid retention symptoms [17]. In contrast, although no study has yet demonstrated that 'a preference for salty food' is associated with premenstrual disorders, decreasing salt intake in the diet has been one of the dietary treatments of premenstrual disorders [23]. In this study, a correlation was observed between 'a preference for salty food' and school absenteeism. We suggest that body water is retained when salty food is eaten, which may lead to physical symptoms such as headaches, weight gain, or edema. We previously demonstrated that physical premenstrual symptoms were related to the high frequency of school absenteeism, and this may be the reason for the higher OR of 'a preference for salty food' than those of other risk factors in lifestyle habits. On the other hand, a preference for salty food may be an aspect of western eating habits, including the daily consumption of junk food and snacks. Eating habits have become more western over the past several decades in Japan, and younger generations consume larger amounts of salty snacks and junk food than older generations [24]. The results from this study may increase awareness that eating habits and increasing consumption of salty snacks or junk food may have a prominent impact on the gynecological health of Japanese women, nevertheless, further investigation is required.

Medications such as serotonin selective reuptake inhibitors (SSRIs) have been the first choice to treat severe PMS and PMDD in adults. However, data on adolescents is lacking. Because SSRIs are difficult to prescribe to adolescents, other approaches towards stress, diet, and exercise may be important. Stress and a poor understanding of the dynamic changes occurring in adolescence may play a 
Table 5 Multivariate analysis of risk factors for school absenteeism due to premenstrual symptoms

\begin{tabular}{lll}
\hline Risk factors & OR & $95 \% \mathrm{Cl}$ \\
\hline Background and lifestyle risk factors & & \\
Age (+1 age) & 1.34 & $1.01-1.79$ \\
Regular exercise habits & 0.73 & $0.46-1.16$ \\
Prefer sweets & 1.04 & $0.60-1.83$ \\
Prefer salty food & $1.89^{\mathrm{a}}$ & $1.20-2.98$ \\
Drink coffee & 1.14 & $0.70-1.85$ \\
Premenstrual symptoms (over moderate severity) & & \\
Depressed mood & 1.37 & $0.73-2.53$ \\
Anxiety or tension & 0.99 & $0.53-1.85$ \\
Tearful & 1.28 & $0.68-2.42$ \\
Anger or irritability & 1.58 & $0.86-2.90$ \\
Decreased interest in work, home, or social activities & 1.83 & $0.99-3.40$ \\
Difficulty concentrating & 1.12 & $0.63-1.97$ \\
Fatigue or lack of energy & 1.61 & $0.91-2.85$ \\
Overeating or food cravings & 0.85 & $0.5-1.43$ \\
Insomnia or hypersomnia & $2.27^{\mathrm{a}}$ & $1.46-4.17$ \\
Feeling overwhelmed & 0.79 & $0.40-1.55$ \\
Physical symptoms & $2.24^{\mathrm{a}}$ & $1.37-3.66$
\end{tabular}

Induced interference with work, usual activities, or relationships (over moderate severity)

Work efficiency or productivity, home responsibility $\quad 1.82 \quad 0.85-3.90$

Social life activities

$2.71^{\mathrm{a}} \quad 1.31-5.59$

Relationships with coworkers or family

$1.26 \quad 0.69-2.29$

OR odds ratio, $95 \%$ Cl $95 \%$ confidence interval, ${ }^{\text {astatistically significant }}$

role in the development of premenstrual disorders. Therefore, education on the effects of ovarian hormones and menstrual cycles on emotional and physical aspects may be helpful to reduce stress related to menstruation, increase awareness, and increase the predictability of menstruation-related problems [6]. Chau et al. reported a significant improvement in knowledge and PMS scores after an educational program for teenage girls [25]. Moreover, guiding school girls with exercise and proper eating habits, including the reduced consumption of salty snacks or junk food, may lead to an improvement in physical premenstrual symptoms, which consequently may improve the school and social life qualities of adolescents.

\section{Conclusions}

In total, $11.9 \%$ of Japanese female high school students were absent from school for more than 1 day per month due to premenstrual symptoms. Premenstrual symptoms, such as 'insomnia or hypersomnia' and 'physical symptoms such as tender breasts, feeling bloating, headache, joint or muscle pain, and weight gain' were risk factors for school absenteeism. In addition, 'a preference for salty food' and 'lack of regular exercise' were risk factors of absence. Considering the difficulty of medical treatment in adolescents, education on proper exercise and eating habits is important and may lead to an improvement in premenstrual symptoms and the life qualities of adolescents.

\section{Abbreviations}

Cl: confidence interval; OR: odds ratio; PMDD: premenstrual dysphoric disorder; PMS: premenstrual syndrome; PSQ: premenstrual symptoms questionnaire; SD: Standard deviation; SSRI: serotonin selective reuptake inhibitors.

\section{Competing interests}

The authors declare that they have no competing interests.

\section{Author's contributions}

MT analyzed the data and wrote the manuscript. TT designed this study and the epidemiologic study at a high school in Sendai and helped draft the manuscript. YM performed the statistical analysis. SK participated in the design and coordination of this study and the epidemiologic study at a high school in Sendai. NY obtained funding for the study and helped draft the manuscript. All authors read and approved the final manuscript.

\section{Acknowledgements}

The authors thank Miho Hayasaka for her help in data collection, and all the girls who participated in this study.

\section{Author details}

'Department of Obstetrics and Gynecology, Tohoku University Graduate School of Medicine, 1-1 Seiryocho, Aobaku, Sendai, Japan. ${ }^{2}$ Division of Women's Health, Research Institute of Traditional Asian Medicine, Kindai University School of Medicine, 377-2 Ohno-Higasi, Osaka-Sayama, Osaka 589-8511, Japan. ${ }^{3}$ Graduate Medical Education Center, Tohoku University Hospital, 1-1 Seiryocho, Aobaku, Sendai, Japan. ${ }^{4}$ Gynecology Clinic Koga, 2-5-12 Kashiwagi, Aobaku, Sendai, Japan.

Received: 29 November 2015 Accepted: 21 April 2016

Published online: 26 April 2016

References

1. Yonkers KA, O'Brein PM, Eriksson E. Premenstrual syndrome. Lancet. 2008; 371:1200-10.

2. Borenstein JE, Dean BB, Endicott J, Wong J, Brown C, Dickerson V, et al. Health and economic impact of the premenstrual syndrome. J Reprod Med. 2003;48(7):515-24

3. American Psychiatric Association. Diagnostic and Statistical Manual of Mental Disorders, Fifth Edition (DSM-V). Arlington: American Psychiatric Association; 2013.

4. Grady-Weliky TA. Clinical practice. Premenstrual dysphoric disorder. N Engl J Med. 2003;348:433-8.

5. Takeda T, Koga S, Yaegashi N. Prevalence of premenstrual syndrome and premenstrual dysphoric order in Japanese high school students. Arch Womens Ment Health. 2010;13:535-7.

6. Rapkin AJ, Mikacich JA. Premenstrual dysphoric disorder and severe premenstrual syndrome in adolescents: diagnosis and pharmacological treatment. Paediatr Drugs. 2013;15:191-202. doi:10.1007/s40272-013-0018-4.

7. Kitamura M, Takeda T, Koga S, Nagase S, Yaegashi N. Relationship between premenstrual symptoms and dysmenorrhea in Japanese high school students. Arch Womens Ment Health. 2012;15:131-3.

8. Derman O, Kanbur NO, Tokur TE, Kutluk T. Premenstrual syndrome and associated symptoms in adolescent girls. Eur J Obstet Gynecol Reprod Biol. 2004;116:201-6.

9. Steiner M, Peer M, Palova E, Freeman EW, Macdougall M, Soarces CN. The premenstrual symptoms screening tool revised for adolescents (PSST-A): prevalence of severe PMS and premenstrual dysphoric disorder in adolescents. Arch Womens Ment Health. 2011;14:77-81. doi:10.1007/s00737010-0202-2. 
10. Issa BA, Yussuf AD, Olatinwo AW, Ighodalo M. Premenstrual dysphoric disorder among medical students of a Nigerian university. Ann African Med. 2010;9:118-22.

11. Drosdzol A, Nowosielski K, Skrypulec V, Plinta R. Premenstrual disorders in Polish adolescent girls: Prevalence and risk factors. J Obstet Gynaecol Res. 2011;37:1216-21. doi:10.1111/j.1447-0756.2010.01505.x.

12. Penland JG, Johnson PE. Dietary calcium and manganese effects on menstrual cycle symptoms. Am J Obstet Gynecol. 1993;168:1417-23.

13. Rossignol AM. Caffeine-containing beverages and premenstrual syndrome in young women. Am J Public Health. 1985;75:1335-7.

14. Rossignol AM, Bonnlander $\mathrm{H}$, Song L, Phillis JW. Do women with premenstrual symptoms self-medicate with caffeine? Epidemiology. 1991;2:403-8.

15. Rossignol AM, Bonnlander H. Prevalence and severity of the premenstrual syndrome. Effects of foods and beverages that are sweet in sugar content J Reprod Med. 1991;36:131-6.

16. Vishnupriya R, Rajarajeswaram P. Effects of aerobic exercise at different intensities in premenstrual syndrome. J Obstet Gynecol India. 2011;61:675-82. doi:10.1007/s|13224-011-0117-5.

17. Daley A. Exercise and premenstrual symptomatology: a comprehensive review. J Women Health (Larchmt). 2009;18:895-9. doi:10.1089/jwh.2008.1098.

18. Takeda T, Tasaka K, Sakata M, Murata Y. Prevalence of premenstrual syndrome and premenstrual dysphoric disorder in Japanese women. Arch Womens Ment Health. 2006;9:209-12.

19. American Psychiatric Association. Diagnostic and Statistical Manual of Mental Disorders, Fourth Edition (DSM-IV). Arlington: American Psychiatric Association; 1994.

20. Steiner $M$, Macdougall $M$, Brown E. The premenstrual symptoms screening tool (PSST) for clinicians. Arch Women Ment Health. 2003;6:203-9.

21. van Eijk AM, Sivakami M, Thakkar MB, Bauman A, Laserson KF, Coates S, et al. Menstrual hygiene management among adolescent girls in India: a systematic review and meta-analysis. BMJ Open. 2016; doi: 10.1136/ bmjopen-2015-010290

22. Delara M, Ghofranipour F, Azadfallah P, Tavafian SS, Kazemnejad A, Montazeri A. Health related quality of life among adolescent girls with premenstrual disorders: a cross sectional study. Health Qual Life Outcomes. 2012;10:1-5. doi:10.1186/1477-7525-10-1.

23. Pearlstein T. Psychotropic medications and other non-hormonal treatments for premenstrual disorders. Menopause Int. 2012;18:60-4. doi:10.1258/mi.2012.012010.

24. Zahra J, Ford T, Jodrell D. Cross-sectional survey of daily junk food consumption, irregular eating, mental and physical health and parenting style of British secondary school children. Child Care Health Dev. 2014;40: 481-91. doi:10.1111/cch.12068.

25. Chau J, Chang A. Effects of an educational programme on adolescents with premenstrual syndrome. Health Educ Res. 1999;14:817-30.

\section{Submit your next manuscript to BioMed Central and we will help you at every step:}

- We accept pre-submission inquiries

- Our selector tool helps you to find the most relevant journal

- We provide round the clock customer support

- Convenient online submission

- Thorough peer review

- Inclusion in PubMed and all major indexing services

- Maximum visibility for your research

Submit your manuscript at www.biomedcentral.com/submit

CBiomed Central 